\title{
Type 2 diabetes mellitus and microvascular complications 1 year after Roux-en-Y gastric bypass: a case-control study
}

\author{
Alexander D. Miras ${ }^{1} \cdot$ Ling Ling Chuah ${ }^{1} \cdot$ Nofal Khalil $^{2} \cdot$ Alessia Nicotra $^{2}$. \\ Amoolya Vusirikala $^{3}$ - Najah Baqai ${ }^{3}$ - Christopher Graham ${ }^{3}$ - Saranya Ravindra ${ }^{3}$. \\ Gerassimos Lascaratos $^{4}$. Nick Oliver ${ }^{1}$ - Carel W. le Roux ${ }^{1,5}$
}

Received: 22 December 2014 / Accepted: 17 March 2015 /Published online: 18 April 2015

(C) The Author(s) 2015. This article is published with open access at Springerlink.com

\begin{abstract}
Aims/hypothesis We aimed to examine the effects of bariatric surgery on microvascular complications in patients with type 2 diabetes using objective measures.

Methods Prospective case-control study of 70 obese surgical patients with type 2 diabetes undergoing gastric bypass surgery matched for age, sex and duration of diabetes to 25 medical patients treated using international guidelines. Microvascular complications were assessed before and 1218 months after intervention using urine albumin creatinine ratio (ACR) measurements, two-field digital retinal images and peripheral nerve conduction studies (in the surgical group only).

Results Urine ACR decreased significantly in the surgical group but increased in the medical group. There were no significant differences between the surgical and medical groups in the changes in retinopathy. There were no changes in the nerve conduction variables in the surgical group.
\end{abstract}

Alexander D. Miras and Ling Ling Chuah are joint first authors.

Ling Ling Chuah

1chuah@nhs.net

1 Investigative Medicine, Division of Diabetes, Endocrinology \& Metabolism, Imperial College London, London, UK

2 Department of Clinical Neurophysiology, West London Neurosciences Centre, Charing Cross Hospital, London, UK

3 Faculty of Medicine, Imperial College London, London, UK

4 Department of Ophthalmology, Guy's and St Thomas' Hospitals Foundation Trust, London, UK

5 Diabetes Complications Research Centre, UCD Conway Institute, School of Medicine and Medical Science, University College Dublin, Dublin, Ireland
Conclusions/interpretation In the short term, bariatric surgery may be superior to medical care in the treatment of diabetic nephropathy, but not retinopathy or neuropathy.

Keywords Bariatric surgery $\cdot$ Nephropathy $\cdot$ Neuropathy $\cdot$ Obesity $\cdot$ Retinopathy
Abbreviations
ACR Albumin creatinine ratio
RYGB Roux-en-Y gastric bypass
SOS Swedish Obese Subjects study

\section{Introduction}

Bariatric surgery reduces weight and improves glycaemia in the long term. The Swedish Obese Subjects (SOS) study used registry data to show that the incidence of microvascular complications in a subgroup of patients with type 2 diabetes mellitus who underwent predominantly vertical banded gastroplasty was lower than that in patients managed with lifestyle interventions [1]. No study so far has used direct clinical measures in a cohort of patients with type 2 diabetes to assess changes in nephropathy, retinopathy and neuropathy after Roux-en-Y gastric bypass (RYGB) surgery.

\section{Methods}

This prospective case-control study took place in a certified centre of excellence for bariatric surgery. In all, 83 obese patients with type 2 diabetes undergoing RYGB surgery [2], the 'surgical group', were recruited between December 2010 and October 2012. These patients were matched for age, sex and 
duration of diabetes to a 'medical' group of 25 patients who did not undergo surgery but who were treated in the same centre using the ADA and EASD guidelines [3].

$\mathrm{BMI}, \mathrm{HbA}_{1 \mathrm{c}}, \mathrm{BP}$, medication usage and microvascular complications were assessed within the 6 months before and at $12-18$ months after the intervention. Nephropathy was assessed using the mean of two early morning urine albumin creatinine ratio (ACR) measurements. Albuminuria was defined as an ACR value of $\geq 2.5 \mathrm{mg} / \mathrm{mmol}$ in men or $\geq 3.5$ $\mathrm{mg} / \mathrm{mmol}$ in women. Retinopathy was assessed with twofield digital retinal images obtained from the English National Screening Programme for Diabetic Retinopathy and were graded by an independent ophthalmologist who was blinded to the patient clinical information, using a five-stage disease severity classification for diabetic retinopathy from the International Clinical Diabetic Retinopathy and Diabetic Macular Oedema Disease Severity Scales [4]. Improvement or worsening was defined as a decrease or increase of at least two steps in the same grading system, respectively. Peripheral neuropathy was assessed through motor and sensory nerve conduction studies, which were performed with standard techniques of stimulation and recording using a Dantec electromyography system (Copenhagen, Denmark). The motor nerves tested were the common peroneal and tibial, and the sensory nerves tested were the superficial radial and sural.

Descriptive statistics are expressed as mean \pm SEM or median and interquartile range depending on normality distribution or as percentages. Within-group comparisons were made using paired sample Student's $t$ tests or the Wilcoxon matched pairs test depending on normality distribution. The retinal data were categorical and analysed using the $\chi^{2}$ test. Bonferroni correction for multiple comparisons was applied to the neurophysiological measurements. The Pearson methodology was used to test correlations between two variables at a time.

The trial protocols were approved by the West London 2 Research Ethics Committee (reference 10/H0711/69). All participants gave written informed consent and the trials were performed according to the principles of the Declaration of Helsinki.

\section{Results}

In the surgical group, 70 patients completed the study as three patients did not proceed to surgery because they were considered to be of too high surgical risk and 10 patients did not return for all the follow-up testing. All 25 patients in the medical group completed the study. There were no significant baseline differences in sex, age and duration of diabetes between the surgical and medical groups.

BMI, systolic $\mathrm{BP}$ and $\mathrm{HbA}_{1 \mathrm{c}}$ were significantly reduced in the surgical, but not the medical groups at 1 year (Table 1).
Urine ACR decreased from 3.6 (1.7-9.3) $\mathrm{mg} / \mathrm{mmol}$ to 1.7 $(1.0-4.9) \mathrm{mg} / \mathrm{mmol}$ in the surgical group $(p=0.02)$, but increased from $1.8(1.2-5.3) \mathrm{mg} / \mathrm{mmol}$ to $3.6(2.0-10.8)$ $\mathrm{mg} / \mathrm{mmol}$ in the medical group at 1 year $(p=0.03)$. Subgroup analysis of the 30 surgical patients who had preexisting albuminuria at baseline showed a significant improvement from $8.5(5.7-17.4) \mathrm{mg} / \mathrm{mmol}$ to $1.7(1.0-6.1)$ $\mathrm{mg} / \mathrm{mmol}$ at 1 year $(p=0.009)$. Fifteen patients normalised their ACR at 1 year. Of the 43 patients who had normal baseline ACR, five developed albuminuria at 1 year post-surgery. By contrast, ACR did not change significantly in the subgroup of the 10 medical patients with pre-existing albuminuria $(p=0.43)$. All of these patients still had albuminuria post-intervention, while out of the 14 patients without albuminuria pre-intervention, five developed albuminuria post-intervention. A $\chi^{2}$ test showed that there was a significant difference between the surgical and medical group for the remission of albuminuria $(p=0.0009)$, but not for the new incidence of albuminuria over the 1 year period $(p=0.10)$.

In the group of 56 surgical patients with complete retinal photograph datasets, 17 had no diabetic retinopathy, 32 had mild to moderate non-proliferative diabetic retinopathy, six had severe non-proliferative diabetic retinopathy, and one had proliferative diabetic retinopathy. At 1 year after surgery, 44 patients $(78 \%)$ had no change, six $(11 \%)$ had improved by at least two steps and six (11\%) deteriorated by at least two steps in retinopathy severity grading. Of the six patients who deteriorated, one required photocoagulation treatment postsurgery. This patient had mild to moderate non-proliferative diabetic retinopathy at baseline. The other five patients had no retinopathy at baseline.

In the medical group, nine participants had no retinopathy, nine had mild to moderate non-proliferative diabetic retinopathy, two had severe non-proliferative diabetic retinopathy and one had proliferative diabetic retinopathy. After intervention, 17 patients $(81 \%)$ experienced no change; one patient $(5 \%)$ improved by at least two steps and three (14\%) deteriorated by at least two steps in the retinopathy severity grading scale. One patient required photocoagulation at follow-up. There were no significant differences between the surgical and medical groups in the rates of patients who deteriorated or improved after intervention ( $p=0.70$ and $p=0.67$, respectively).

In all, 54 participants in the surgical group underwent nerve conduction studies and there were no clinically significant changes in any of the nerve conduction variables at 1 year.

There were no significant correlations between changes in ACR and change in $\mathrm{HbA}_{1 \mathrm{c}}(r=-0.046, p=0.75)$, BMI $(r=$ $-0.058, p=0.70)$, and systolic $(r=-0.079, p=0.61)$ and diastolic BP $(r=0.042, p=0.79)$ in either group.

There were significant reductions in use of glucoselowering and BP-lowering medications in the surgical, but not the medical group at 1 year follow-up (Tables 1 and 2). 


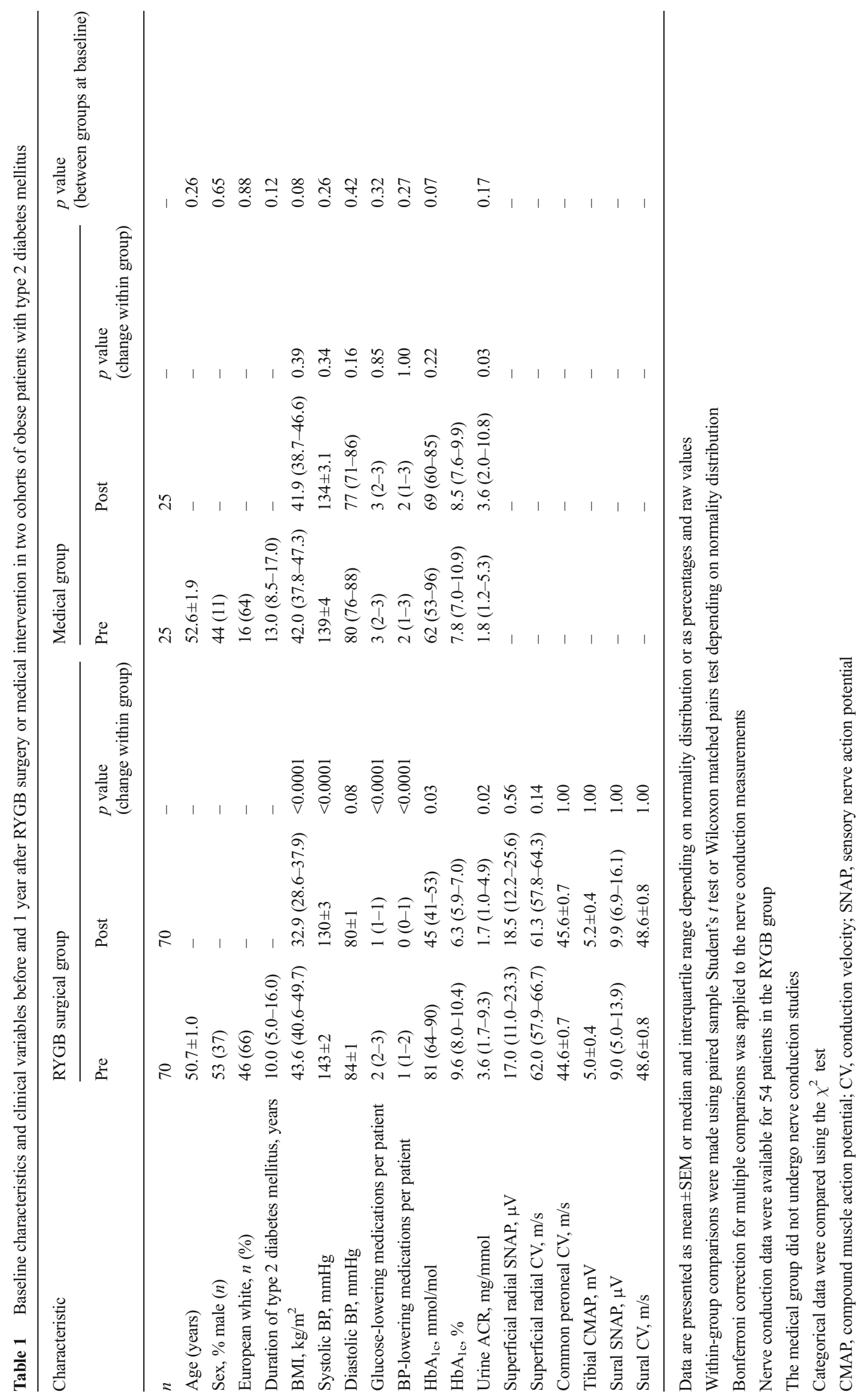


Table 2 Medication classes before and 1 year after RYGB surgery or medical intervention in two cohorts of obese patients with type 2 diabetes mellitus

\begin{tabular}{llllll}
\hline Medication & \multicolumn{2}{c}{$\begin{array}{l}\text { RYGB surgical group } \\
(n=70)\end{array}$} & & \multicolumn{2}{l}{$\begin{array}{l}\text { Medical group } \\
(n=25)\end{array}$} \\
\cline { 2 - 3 } \cline { 5 - 6 } \cline { 5 - 6 } & Pre & Post & & Pre & Post \\
\cline { 1 - 3 } Patient use of glucose-lowering medications classes, & $n(\%)$ & \\
Metformin & $58(83)$ & $41(59)$ & $19(76)$ & $18(72)$ \\
Sulphonylurea & $15(21)$ & $0(0)$ & & $7(28)$ & $9(36)$ \\
Pioglitazone & $4(6)$ & $0(0)$ & & $3(12)$ & $2(8)$ \\
DPP4i/GLP-1 & $26(37)$ & $1(1)$ & $10(40)$ & $13(52)$ \\
Insulin & $46(66)$ & $20(29)$ & $15(60)$ & $17(68)$ \\
Patient use of BP-lowering medications, & $n(\%)$ & & \\
ACEi/A2B & $48(69)$ & $26(37)$ & $16(64)$ & $18(72)$ \\
Calcium channel blocker & $18(26)$ & $15(21)$ & $1(4)$ & $2(8)$ \\
Alpha blocker & $7(10)$ & $2(3)$ & $8(32)$ & $7(28)$ \\
Diuretics & $18(26)$ & $2(3)$ & $6(24)$ & $6(24)$ \\
Beta blocker & $6(9)$ & $5(7)$ & $6(24)$ & $7(28)$ \\
\hline
\end{tabular}

$n$ refers to the number of patients in the cohort that were on the class of medications

A2B, angiotensin receptor 2 blocker; ACEi, ACE inhibitors; DPP4i, dipeptidyl peptidase-4 inhibitors; GLP-1, glucagon-like peptide 1

\section{Discussion}

In this prospective case-control study, we used direct and objective markers of nephropathy, retinopathy and neuropathy and found that after 1 year, RYGB surgery may be superior to medical treatments for nephropathy, but not for retinopathy. In studies of intensive medical management, substantial and rapid reductions in blood glucose, similar to those that take place after RYGB, were associated with paradoxical deterioration of microvascular complications [5]. It was reassuring to observe that the rates of patients experiencing worsening in retinopathy in the RYGB group was not in excess to those observed in patients receiving medical care based on international guidelines. The stability of neuropathy after 1 year was also reassuring.

We, and others, have previously shown that albuminuria improves early after bariatric surgery $[6,7]$ and this may translate to better long-term renal outcomes, like those observed by the SOS study [1]. Retinal and neuronal function may take longer than 1 year after bariatric surgery to show clinically meaningful changes. This is reminiscent of the very slow improvements in neuropathy after pancreatic or islet cell transplantation in patients with type 1 diabetes mellitus [8]. The mechanisms of improvement in albuminuria after bariatric surgery remain unclear. We expect that despite the lack of correlations, the reductions in blood pressure, $\mathrm{HbA}_{1 \mathrm{c}}$ and $\mathrm{BMI}$ contributed to the observed reductions in $\mathrm{ACR}$.
Our study is limited by the small sample size, the nonrandomised design, short follow-up and lack of information on peripheral neuropathy for the medically treated patients. However, our findings could be used to power larger randomised controlled trials that are necessary in this field. We cannot exclude that some patients may have had an acute deterioration in retinopathy that occurred before 12 months and then partially recovered. We are, however, reassured that the surgical patients who were all followed at 3, 6 and 9 months after surgery did not complain of deteriorating vision. Moreover, the retinopathy findings at 1 year were also reassuring.

In summary, we have shown that compared with a group of patients receiving best medical care, patients treated with bariatric surgery experience substantial reductions in albuminuria. In the short term, bariatric surgery may be superior to medical care in the treatment of diabetic nephropathy, but not retinopathy or neuropathy.

Funding The Section is funded by grants from the Medical Research Council (MRC), the Biotechnology and Biological Sciences Research Council (BBSRC), the National Institute for Health Research (NIHR), an Integrative Mammalian Biology (IMB) Capacity Building Award, and a FP7-HEALTH-2009-241592 EuroCHIP grant and is supported by the NIHR Imperial Biomedical Research Centre Funding Scheme. ADM has received funding from an MRC Clinical Training Fellowship, MRC Centenary Award and an NIHR Clinical Lectureship. CWIR has received funding from the Science Foundation Ireland (12/YI/B2480) and the Moulton Foundation UK. We would like to thank the staff of the Imperial Weight Centre for their tireless efforts in looking after these patients.

Access to research materials Data can be obtained through application to the corresponding author.

Duality of interest The authors declare that there is no duality of interest associated with this manuscript.

Author contributions LLC contributed to the conception and design of the study, acquired, analysed and interpreted data, and drafted and revised the article. ADM and CWIR contributed to the conception and design of the study, analysed and interpreted data and revised the article. $\mathrm{NK}, \mathrm{AN}, \mathrm{GL}, \mathrm{NO}$ researched data and contributed to the interpretation of the data and revised the article. AV, NB, CG, SR researched data, and revised the article. All authors approved the final version of the manuscript. CWIR is the guarantor of this work.

Open Access This article is distributed under the terms of the Creative Commons Attribution 4.0 International License (http://creativecommons.org/ licenses/by/4.0/), which permits unrestricted use, distribution, and reproduction in any medium, provided you give appropriate credit to the original author(s) and the source, provide a link to the Creative Commons license, and indicate if changes were made.

\section{References}

1. Sjostrom L, Peltonen M, Jacobson P et al (2014) Association of bariatric surgery with long-term remission of type 2 diabetes and with microvascular and macrovascular complications. JAMA 311:2297-2304 
2. Olbers T, Lonroth H, Fagevik-Olsen M, Lundell L (2003) Laparoscopic gastric bypass: development of technique, respiratory function, and long-term outcome. Obes Surg 13:364-370

3. Nathan DM, Buse JB, Davidson MB et al (2009) Medical management of hyperglycaemia in type 2 diabetes mellitus: a consensus algorithm for the initiation and adjustment of therapy: a consensus statement from the American Diabetes Association and the European Association for the Study of Diabetes. Diabetologia 52: $17-30$

4. Wilkinson CP, Ferris FL 3rd, Klein RE et al (2003) Proposed international clinical diabetic retinopathy and diabetic macular edema disease severity scales. Ophthalmology 110:16771682
5. Dahl-Jorgensen K, Brinchmann-Hansen O, Hanssen KF, Sandvik L, Aagenaes O (1985) Rapid tightening of blood glucose control leads to transient deterioration of retinopathy in insulin dependent diabetes mellitus: the Oslo study. BMJ (Clin Res Ed) 290:811-815

6. Miras AD, Chuah LL, Lascaratos G et al (2012) Bariatric surgery does not exacerbate and may be beneficial for the microvascular complications of type 2 diabetes. Diabetes Care 35, e81

7. Iaconelli A, Panunzi S, de Gaetano A et al (2011) Effects of biliopancreatic diversion on diabetic complications: a 10-year follow-up. Diabetes Care 34:561-567

8. Vantyghem MC, Quintin D, Caiazzo R et al (2014) Improvement of electrophysiological neuropathy after islet transplantation for type 1 diabetes: a 5-year prospective study. Diabetes Care 37:e141-e142 